\title{
Differential effects of delay of reinforcement on acquisition of affective and instrumental responses
}

\author{
M. R. D'AMATO and W. R. SAFARJAN \\ Rutgers University, New Brunswick, New Jersey 08903
}

\begin{abstract}
Thirsty rats were allowed to choose between a striped arm of a T-maze that contained water and a black arm that contained a saccharin solution. After each exposure to the saccharin solution, half of the animals received a lithium chloride injection 1 min later and half received the same injection $30 \mathrm{~min}$ later. Comparable saline injections at these same delays were given after each exposure to the striped, water, arm. The results from saccharin aversion, spatial aversion, and instrumental learning tests generally supported the hypothesis that delayed reinforcement has a substantially greater negative impact on the acquisition of an instrumental response than it has on affective conditioning.
\end{abstract}

We have recently found that, with proper parameter values, monkeys are capable of developing a preference for a spatial location (a distinctive arm of a T-maze) in a single trial and with a 30 -min delay separating exposure to that location and subsequent food reinforcement (D'Amato \& Buckiewicz, 1980). Although there have been previous reports of one-trial appetitive conditioning in animals (e.g., Albert \& Mah, 1972), to our knowledge this is the first report of such conditioning in which a substantial delay of reinforcement was employed. Moreover, it appears that similar results are obtainable with rats, with delays of up to $2 \mathrm{~h}$ (Safarjan \& D'Amato, Note 1). These findings, in conjunction with the many taste aversion studies which have shown one-trial, long-delay conditioned aversion in rats and primates (cf. Barker, Best, \& Domjan, 1977), suggest that affective responses, whether positive or negative, whether based on exteroceptive cues or taste, are conditionable within a single trial and with substantial delays intervening between termination of the CS and onset of the UCS.

Although the boundaries of this generalization remain to be established by future research, the results already in hand prompt the following question. If positive affective responses (i.e., preference for a spatial location and the like) are so readily established and so resistant to delay of reinforcement, why is it that even a modest delay of reward causes such interference with the acquisition of instrumental behavior, viz., spatial and visual discrimination learning? It is true that even with conditioned reinforcement cues

This research was supported by National Science Foundation Grant 78-24644. Reprints may be obtained from M. R. D'Amato, Psychology Department, Busch Campus, New Brunswick, New Jersey 08903. W. R. Safarjan is now at Porterville State Hospital, P.O. Box 2000, Porterville, California 93257. largely eliminated, rats can learn a spatial discrimination response rather efficiently with delays of a few minutes, a fact that has been known for some time (e.g., Wolfe, 1934). However, the evidence for spatial discrimination learning with longer delays, say in the neighborhood of $20 \mathrm{~min}$ or longer, is scarce and not yet compelling (Lett, 1975). The situation is far worse in the case of visual discrimination learning, in which there is even some question as to whether rats can learn a simple black-white discrimination when reward is delayed for as little as $1 \mathrm{~min}$ (Roberts, 1976, 1977; but see Lett, 1978). Although monkeys are capable of efficient visual discrimination learning with delays in this order of magnitude (D'Amato \& Cox, 1976), we were unable to obtain evidence of visual discrimination learning with delays of $16 \mathrm{~min}$ (D'Amato \& Cox, Note 2; cf. Riesen, 1940). It appears that if appropriate affective responses are conditioned in these situations, some factor or factors inhibit expression of this conditioning in terms of the target instrumental behavior.

The aim of the present experiment was to assess, within the same experimental situation, this apparent differential sensitivity of affective and instrumental learning to the deleterious effects of delay of reinforcement. By concurrently assessing within the same experimental situation the rate of affective and instrumental conditioning, one can determine whether delay of reinforcement interferes with the acquisition of instrumental behavior because it inhibits conditioning of the appropriate affective response or whether the fault lies elsewhere.

Because we wished to evaluate more than a single affective response, we enlisted the taste aversion paradigm to provide the source of reinforcement for conditioning of the affective and instrumental responses. 
In brief, thirsty rats were given a water reward in one arm of a T-maze and a saccharin solution in the other, distinctively colored, arm. Following each saccharin solution reward, some animals were poisoned with lithium chloride shortly thereafter while others were poisoned $30 \mathrm{~min}$ later. Three dependent variables were assessed: aversion for the saccharin solution (measured by consumption of saccharin solution vs. water), aversion for the arm of the maze associated with the saccharin solution (measured by spatial preference tests), and acquisition of the instrumental response of choosing the arm that contained water (measured by choice behavior). The hypothesis under test was that delay of reinforcement, poisoning in this case, would have a greater impact on acquisition of the instrumental response than it would have on acquisition of the taste or the spatial aversion.

\section{METHOD}

\section{Subjects}

The 32 naive female albino rat (ARS Sprague-Dawley, Madison, Wisconsin) subjects were maintained on a 12-h light-dark cycle with free access to food. Six days before pretraining, the animals were housed individually and access to water was restricted to 10 min per day. Each subject was weighed daily and handled for several minutes a day. The animals were about 85 days old and weighed between 172 and $204 \mathrm{~g}$ at the time of pretraining.

\begin{abstract}
Apparatus
The conditioning and testing apparatus was a small T-maze (14 cm in height), constructed of wood. The two arms (each $14 \mathrm{~cm}$ wide and $40.5 \mathrm{~cm}$ long) were separated by an unpainted stem (19 cm wide, $33 \mathrm{~cm}$ long), from which a startbox (19 cm long) was partitioned by an opaque guillotine door. A transparent guillotine door was located at the entrance of each arm. The walls and floor of the left arm were vertically striped with alternating black and white $1.3-\mathrm{cm}$ bars; the right arm was black. A glass drinking tube could be positioned at the end of each arm to dispense either tap water (in the left, striped arm) or a .1\% $(w / v)$ saccharin solution (in the right, black, arm). Both fluids were maintained at room temperature and were changed daily. The drinking tubes (graduated in milliliters) containing the fluids were located outside the $\mathrm{T}$-maze, with the glass spout protruding through the end wall of the arm. The subjects could be placed into either arm or into the startbox through transparent doors, which served as the "ceiling" of the maze. For the left (striped) arm, the ceiling was striped with $1.3-\mathrm{cm}$ white bars, spaced $3.9 \mathrm{~cm}$ apart. The floor of the maze was covered with clear plastic matting.

Two 25-W bulbs, situated about $1 \mathrm{~m}$ above each arm and equidistant from the center of the stem, served as the main source of illumination. Continuous white noise, about $75 \mathrm{~dB}$, was used to mask extraneous sounds.
\end{abstract}

\section{Procedure}

Pretraining. In Session 1 of pretraining, each subject was confined for $5 \mathrm{~min}$ in the striped arm of the T-maze, which contained water, and fluid consumption was recorded. The subject was then returned to the colony room, where, $30 \mathrm{~min}$ after the last animal was run, it received 10 min access to water. In Session 2, the subjects were confined for $5 \mathrm{~min}$ in the black arm of the T-maze, which contained saccharin solution, and the amount of saccharin consumed was recorded. In Session 3, baseline spatial preference was evaluated with neither fluid present in the T-maze. The animal was placed in the startbox, and about $30 \mathrm{sec}$ later the startbox door was raised, allowing the animals to explore the maze. A subject was judged to have entered an arm when all four feet crossed a line that marked the entry into the arm. It was judged to have left the arm when all four feet crossed over the entry line into the stem. Timing for the 5-min preference test began with the animal's first entry into an arm. At the end of $5 \mathrm{~min}$, the subject was removed from the T-maze and returned to the colony room. Watering conditions were the same as those in Sessions 1 and 2. A spatial preference score was obtained for each animal by dividing the amount of time spent in the black (right) arm by the amount of time spent in both arms, multiplied by 100 (time spent in the stem was excluded). The three pretraining sessions occurred on successive days.

Training (Sessions 4-19). The animals were assigned to one of four groups (eight subjects each), equated with regard to the baseline preference scores obtained during Session 3. For two groups of animals, Groups $\mathrm{U} 1$ and $\mathrm{U} 30$ (uncontrolled intake), the animals were allowed to drink freely during the 5 min that they were confined in an arm of the T-maze following a free or forced choice. In both groups, a free-choice trial was followed by a forced choice to the side opposite to that chosen on the previous trial. There was a total of 16 training trials, one per session. On a free-choice trial, the subject was carried to the experimental room and placed in the startbox of the T-maze; about $30 \mathrm{sec}$ later the startbox door was raised. After the animal entered an arm, the guillotine door of the chosen arm was lowered; the animal was confined to the arm for $5 \mathrm{~min}$, during which it was free to drink tap water (available in the left, striped arm) or saccharin solution (available in the right, black arm). Initially, the 5 -min period was timed from the first contact with the drinking tube, but on Trial 4 timing commenced upon entry into the arm. At the end of the 5-min period, the animal was returned to the colony room. After each trial, the T-maze was cleaned with a mild deodorizer. The training procedure was identical on forced trials, except that the door leading to the arm selected on the preceding trial was lowered, forcing the animal to the opposite arm (and fluid). Alternating free and forced trials insured that each subject had an equal number of exposures to both arms and to both fluids.

Following each exposure to the saccharin solution in the black arm, whether the result of a free or a forced trial, all subjects were poisoned with $4-\mathrm{ml} / \mathrm{kg}$ injection (ip) of isotonic $(.15 \mathrm{M})$ lithium chloride (.6 mEq). For animals in Group U1, the injection was given immediately after the animal was returned to the colony room, which was approximately $1 \mathrm{~min}$ following removal from the T-maze. For the subjects in Group U30, the lithium chloride injection was given $30 \mathrm{~min}$ after the animals were returned to the colony room following exposure to the saccharin solution. To compensate for possible effects of the injection itself, an isotonic saline solution, comparable in quantity to the lithium chloride injection, was administered 1 or $\mathbf{3 0}$ min (for Groups U1 and U30, respectively) after trials in which the animal was exposed to the water (striped) arm of the T-maze.

Because of the poisoning contingency associated with ingestion of the saccharin solution, it was to be expected that as training progressed the animals in Groups U1 and U30 would consume less saccharin solution than water. Consequently, Groups $\mathrm{Cl}$ and C30 (controlled intake), which were otherwise treated in exactly the same way as the corresponding Groups U1 and U30, had their consumption of water in the striped arm adjusted so as to make it comparable to the amount of saccharin solution consumed in the black arm. This was accomplished by removing the drinking tube containing tap water as soon as the animal had consumed an amount of water approximately equal to the amount of saccharin solution consumed during the previous exposure to saccharin.

To mitigate possible cumulative poisoning effects, training sessions were conducted every other day. On nontraining days, the animals received the usual 10 -min water ration but were otherwise left undisturbed.

Aversion for the black, saccharin, arm was assessed by two 5-min spatial preference tests, one administered 2 days after Train- 
ing Trial 4 and the second, 2 days after Training Trial 12. These "Trial 4" and "Trial 12"' preference tests were conducted in exactly the same manner as the baseline preference test administered during pretraining (Session 3), and, as with the latter test, no fluid was available in the T-maze. To reduce possible extinction effects that might result from a preference test, a free-choice training trial was run immediately after each preference test. Thus, as soon as the preference test was completed, the animal was confined to the startbox for about $1 \mathrm{~min}$ while the arms of the T-maze were cleaned and the drinking tubes were inserted into the arms of the maze. The startbox door was then raised and the animal allowed to make a choice, which was subsequently followed by either the saline or the lithium chloride injection, depending upon whether the animal chose the striped (water) or the black (saccharin) arm. Following so closely upon the preference test, the animal's response on the free-choice trial was very likely influenced by its behavior on the preference test. Therefore, the response outcome on these training trials ( 5 and 13 ) was taken to be the animal's initial arm entry on the preference test that immediately preceded the free-choice trials. This procedure seemed to provide an uncontaminated free-choice outcome while at the same time reducing the possible extinction effects of the preference tests.

Controls for spatial aversion. An additional eight subjects were included to provide an assessment of the degree of preference for the black (saccharin) arm that would have prevailed on the Trial 4 preference test had the animals not been poisoned. These subjects, which were of the same age and sex and from the same vendor as the 32 experimental animals described previously, were run after the experiment proper was completed. They received the same three pretraining sessions as described above. The four training trials that followed differed from those given to the experimental animals in that no injections of either lithium chloride or saline were administered. On Training Trials 1 and 2, the animals were allowed to drink freely during the 5-min access to water or to saccharin. However, on Trials 3 and 4 , to make their intake of water and saccharin comparable to that of the experimental subjects, water intake was restricted to the mean amount of water consumed by the 32 experimental subjects on corresponding trials $(4.9 \mathrm{ml})$ and consumption of saccharin was similarly restricted $(4.3 \mathrm{ml})$. Apart from these modifications, the animals were treated in precisely the same fashion as the 32 experimental subjects. Because reduction of saccharin consumption following poisoning by lithium chloride is so well documented (cf. Barker et al., 1977), there was no need for parallel saccharinaversion control subjects.

\section{RESULTS}

\section{Conditioned Affective Responses}

Taste aversion. The strength of the conditioned aversion developed for the saccharin solution was evaluated in the uncontrolled-intake groups (U1 and U30) by a saccharin preference score derived from each successive pair of training trials, which, because free trials alternated with forced trials, always included a 5-min exposure to saccharin and a 5-min exposure to water. For any given pair of trials, saccharin preference was defined as the amount of saccharin consumed on that pair of trials divided by the total consumption of water and saccharin on the two trials (multiplied by 100). Figure 1 presents, for Groups U1 and U30, the eight saccharin preference scores generated by the 16 training trials. It is clear from the figure that despite a considerable disparity in delay of reinforcement, there was little difference between Groups $\mathrm{U} 1$ and $\mathrm{U} 30$ with regard to the aver-

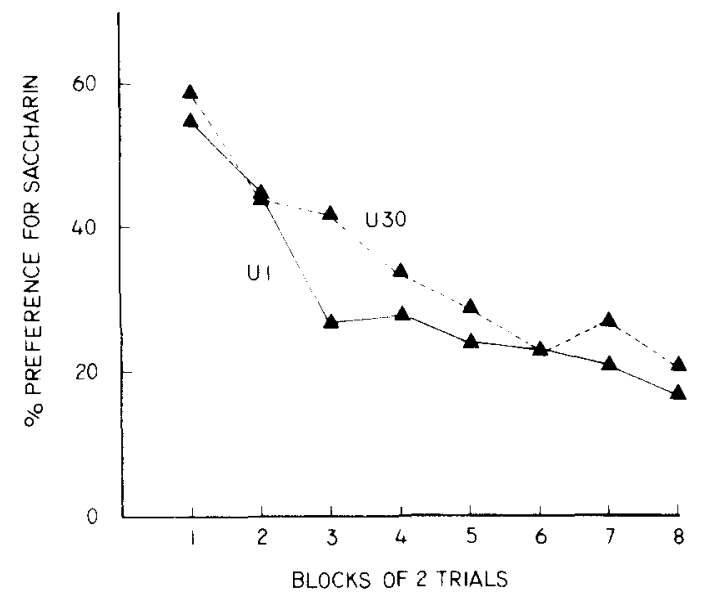

Figure 1. Percent preference for the saccharin solution in the uncontrolled-intake groups based on successive pairs of training trials, which always included a 5-min exposure to saccharin and a 5-min exposure to water. A lithium chloride injection was administered $30 \mathrm{~min}$ after each saccharin exposure in Group U30 and after $1 \mathrm{~min}$ in Group U1.

sion developed for the saccharin solution. This impression was verified by an analysis of variance (ANOVA), which produced a significant Trials effect $[F(7,98)=18.33, p<.001]$ but nonsignificant effects for Groups and for the Groups by Trials interaction $(\mathrm{Fs}<1)$.

Because consumption of water and saccharin solution was controlled in Groups $\mathrm{Cl}$ and $\mathrm{C} 30$, the saccharin preference scores of these groups have no bearing on the aversion developed for saccharin. It might be noted, however, that the mean saccharin "preference" score over Trials 3-16 was 47\% for Group $\mathrm{C} 1$ and $54 \%$ for Group C30, indicating that, as intended, each group consumed comparable amounts of water and saccharin solution.

Figure 2 presents the amount of saccharin solution consumed in the four groups expressed as a percentage of saccharin consumption on the first pair of trials (which preceded the first poisoning experience). Groups U1 and U30 produced similar ingestion patterns with regard to this measure of saccharin consumption; however, there was a considerable disparity in the amount of saccharin consumed in the controlledintake groups ( $\mathrm{C} 1$ and $\mathrm{C} 30$ ). An ANOVA applied to the data of Figure 2 revealed significant effects for Trials $[F(6,168)=18.59, \mathrm{p}<.001]$, Delay $[\mathrm{F}(1,28)=$ $7.77, \mathrm{p}<.01]$, and the Delay by Intake interaction $[F(1,28)=5.28, p<.03]$. The last result reflects the fact that whereas the difference in saccharin consumption between Groups $\mathrm{Cl}$ and $\mathrm{C} 30$ was significant $[\mathrm{t}(28)=3.60, \mathrm{p}<.001]$, the difference between the corresponding uncontrolled-intake groups was not $[\mathrm{t}(28)=.35]$.

Spatial aversion. Aversion for the arm in which saccharin appeared (black) was evaluated by 5 -min spatial preference tests administered after 4 and after 
12 training trials. These data, as well as the baseline preference scores obtained on Pretraining Session 3 (which preceded the first training trial), are shown in Figure 3 in terms of preference for the black side. As with the saccharin preference measure, preference for the black arm was defined as time spent in that arm divided by total time spent in both arms (multiplied by 100). Because assignment of groups was based on the baseline spatial preference scores, the latter are quite comparable across the four groups. Baseline preference for the black arm averaged over the 32 subjects, $59.5 \%$, was significantly greater than chance $(50 \%)$ expectation $[\mathrm{t}(31)=7.48, \mathrm{p}<.001]$, indicating an initial preference for the black, saccharin arm. However, after four training trials, in only two of which saccharin was paired with lithium chloride, all four groups changed their preference from the black to the striped arm of the T-maze. Because the Trial 4 preference test results were so comparable across the four groups, statistical evaluation was based on the overall mean preference for the black side, $40.2 \%$, which was significantly below chance expectation [t $(31)=6.72, p<.001]$. Of the 32 animals in the four groups, 30 decreased their preference for the black arm on the Trial 4 preference test. Aversion for the black arm was even greater on the Trial 12 test (35.9\%), but, as evaluated by a 2 (tests) by 2 (delays) by 2 (intake) ANOVA, neither the Trial 4 vs. Trial 12 comparison nor any of the other comparisons was significant.

Spatial preference was also evaluated in terms of the absolute amount of time spent in the black arm of the T-maze. This measure produced essentially the same pattern of results as the relative preference mea-
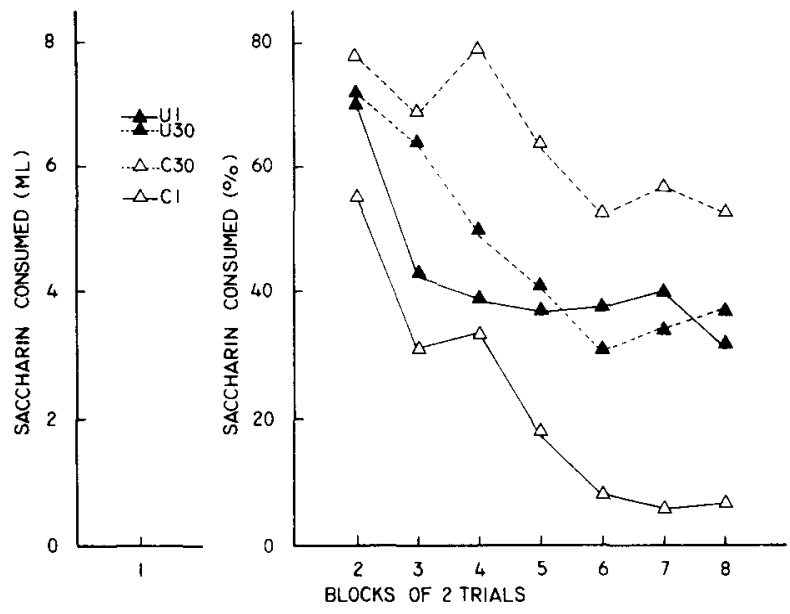

Figure 2. The data in the left portion of the figure give the volume of saccharin consumed in the four groups during the first pair of trials, which preceded the first lithium chloride injection; the differences among the four groups were not significant. The right side of the figure shows the amount of saccharin solution consumed during subsequent pairs of training triak, expressed as a percentage of saccharin consumption on the first pair of trials. The experimental conditions were controlled (C) vs. uncontrolled (U) intake and delay of poisoning, 1 or $30 \mathrm{~min}$.

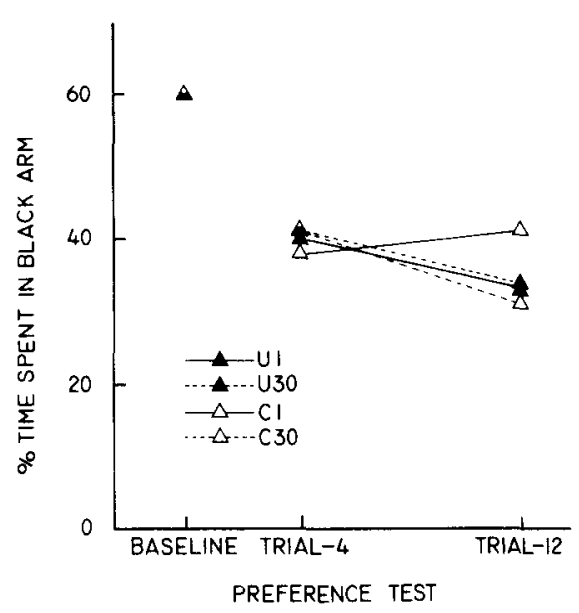

Figure 3. Percent preference for the black, saccharin, arm of the T-maze in the four groups of the experiment during baseline, Trial 4, and Trial 12 spatial preference tests. No fluid was present in the maze during these tests.

sure. The mean durations of time spent in the black arm during the baseline, Trial 4 , and Trial 12 preference tests were $120.7,87.8$, and $78.8 \mathrm{sec}$, respectively. The decrease from baseline to the Trial 4 test was highly significant $[F(1,28)=83.4, p<.001]$; the Trial 4 vs. Trial 12 difference was nonsignificant, and there were no significant differences among the four groups on any of the tests.

\section{Control Subjects}

Consistent with the experimental animals, the eight control animals evidenced an overall preference for the black, saccharin side during the baseline spatial preference test, which because of the relatively small number of subjects involved, did not reach statistical significance when compared with a chance $(50 \%)$ level [mean $=55.4 \%, t(7)=2.11, .05<p<.10$ ]. In contrast with the experimental animals, on the Trial 4 preference test, the controls increased their preference for the black, saccharin side, which was now highly significant [mean $=68.6 \%, t(7)=5.89, p<.001$ ] Thus, in the absence of the saccharin-poisoning contingency, an increase in preference, rather than an aversion, developed during Trials $1-4$ for the black, saccharin arm of the T-maze.

\section{Instrumental Behavior}

Acquisition of the instrumental response, that is, learning to choose the striped, water side of the maze, was reflected in the eight free-choice trials. Figure 4 presents these data for the combined delay groups in blocks of four trials. It is apparent from the figure that, whereas the 1-min delay groups showed clear signs of learning by the end of the eight free-choice trials, there was no evidence of acquisition in the 30min delay groups. An ANOVA based on the Delay, Intake (Controlled vs. Uncontrolled), and Blocks variables produced a significant result for Delay $[F(1,28)$ $=5.68, p<.03$ ] and a marginally significant $F$ for 


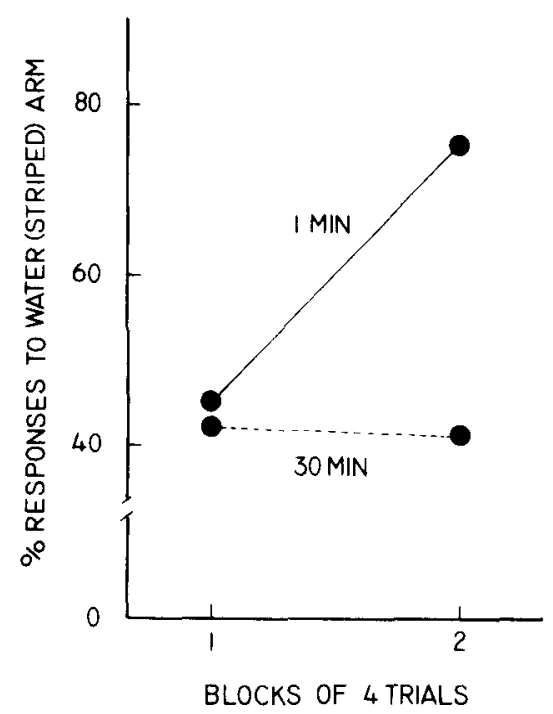

Figure 4. Percent response to the water, striped, arm of the Tmaze averaged across blocks of four free-choice trials. The data are collapsed across the controlled- vs. uncontrolled-intake groups. A lithium chloride injection followed 1 or $30 \mathrm{~min}$ after each choice of the black, saccharin, arm, whereas a comparable saline injection was administered after each choice of the striped, water, arm.

the Delay by Blocks interaction $[F(1,28)=3.92, p<$ .058 . The three-way interaction was not significant. Of the 16 animals in the 1 -min delay groups, 11 increased the number of correct responses achieved in the second block of 4 free-choice trials compared with the first block, three decreased the number of correct responses, and 2 showed no change. As evaluated by a sign test (one tail), the increase in correct responses over the two blocks shown by Groups $\mathrm{C} 1$ and U1 is significant $(\mathrm{p}=.029)$.

In view of the difference in saccharin aversion observed in the two controlled-intake groups $(\mathrm{Cl}$ and $\mathrm{C} 30$ ), it is worth noting that over the last four freechoice trials, the difference in percent correct responses between Groups U1 and U30 was somewhat greater than the difference between Groups $\mathrm{Cl}$ and C30 $(40 \%$ vs. $28 \%)$. Thus, delay of poisoning interfered with acquisition of the instrumental response in both the controlled- and uncontrolled-intake conditions.

\section{DISCUSSION}

The present experiment was motivated by the hypothesis that delay of reinforcement has a more deleterious effect on the acquisition of instrumental behavior than on the conditioning of affective responses. The obtained pattern of results generally supports the hypothesis. With regard to Groups U1 and U30, there was little difference in the degree of aversion developed toward the saccharin solution, either in terms of absolute consumption of saccharin or in terms of relative consumption of saccharin and water. Similarly, the degree of aversion developed for the black (saccharin) arm on the T-maze was quite comparable in the two groups. In contrast, the eight animals of Group U1 averaged $78 \%$ correct responses over the second block of four free-choice trials compared with only $\mathbf{3 8 \%}$ for Group U30. Thus, the 30-min delay of poisoning had little discernible effect on the conditioning of either the saccharin aversion or the spatial aversion in Groups U1 and $\mathrm{U} 30$ but a marked effect on instrumental behavior.

The pattern of results obtained in Groups $\mathrm{Cl}$ and C30 was less favorable to the hypothesis in that there was a substantial difference in the amount of saccharin solution consumed in these groups. Because multiple poisonings were required, we employed a low, isotonic dosage of lithium chloride, which may have been a contributing factor. But dosage alone cannot simultaneously account for the difference obtained in Groups $\mathrm{Cl}$ and $\mathrm{C} 30$ and the lack of a saccharin aversion difference in Groups U1 and U30. Groups C1 and $\mathrm{C} 30$ were included in the experiment as controls for the unequal water and saccharin consumption expected in Groups $\mathrm{U} 1$ and $\mathrm{U} 30$ as the conditioned saccharin aversion took hold. We were concerned that the differential fluid ingestion in favor of tap water would serve as a source of differential immediate (positive) reinforcement for Groups U1 and U30, obscuring possible differences in instrumental learning due to the delay-of-poisoning variable. However, it seems that the effects of the latter variable on instrumental behavior were sufficiently strong to overcome this dampening influence.

Although somewhat remote, a possible reason for the difference in saccharin aversion that emerged between Groups $\mathrm{Cl}$ and $\mathrm{C} 30$ is that development of the discrimination between ingestion of saccharin and subsequent injection of lithium chloride was inhibited in Group C30 because of the similar stomach loads that existed in the controlled-intake groups at the time of the saline and lithium chloride injections. In Group U30, on the other hand, after the first few training trials there was a substantial difference in the amount of fluid consumed in the two arms of the Tmaze, which might have facilitated later association of the lithium chloride injection with ingestion of the saccharin solution. A major problem with this and related explanations is that the difference between Groups $\mathrm{C} 1$ and $\mathrm{C} 30$ is due as much to low saccharin consumption in Group C1 (as compared with the uncontrolled-intake groups) as to relatively high intake in Group C30 (see Figure 2).

Although there was a difference in the saccharin aversion displayed by Groups $\mathrm{C} 1$ and $\mathrm{C} 30$, no such difference appeared with regard to spatial aversion of the black, saccharin side, and in this respect Groups $\mathrm{Cl}$ and $\mathrm{C} 30$ were quite comparable to Groups $\mathrm{U} 1$ and $\mathrm{U} 30$. Turning to the instrumental response, 
the separation between the 1- and 30-min groups was, as already mentioned, no larger in the controlledintake condition than in the uncontrolled condition, indicating that, with respect to its effect on instrumental behavior, the delay variable was equally effective in both conditions.

It would have been desirable to continue training beyond Trial 16, which was our original intention. However, consumption of the saccharin solution had, by Trial 10, dropped so far in some of the animals that on four occasions an animal failed to lick the drinking tube. This posed a serious methodological dilemma with respect to whether the animals should be poisoned on such trials. Omitting the poisoning treatment might result in some extinction of the conditioned spatial aversion, while administration of lithium chloride might have the same effect on the taste aversion. We judged the latter as less likely and therefore maintained the customary poisoning treatment.

No attempt was made in this study to determine whether the aversion for the black arm of the Tmaze was in some way mediated or potentiated by the saccharin aversion. This issue was not directly related to the problem that primarily concerned us, namely, whether the instrumental behavior and the spatial aversion, whatever the source of the latter, differed substantially in the degree of their dependence on the delay-of-poisoning variable. There is reason to believe, however, that the spatial aversion was not completely mediated by the saccharin aversion. The difference in saccharin aversion observed in the controlled-intake groups taken in conjunction with their comparable spatial aversion argues against that possibility. And, as pointed out by Best, Best, and Henggeler (1977), the existence of taste or ingestion-related cues tends to overshadow control by exteroceptive stimuli. These investigators also reported toxicosis-induced aversions to exteroceptive (visual) cues, but with delays shorter than $30 \mathrm{~min}$ and larger numbers of conditioning trials. Although potentiation of visual aversion learning by a taste cue has been reported in birds, the effect seems more the result of enhanced attention to the visual cue than to direct mediation by a taste-aversion association (Lett, 1980; cf. Galef \& Osborne, 1978).

Granting that the present results support the hypothesis that affective responses are less at the mercy of reinforcement delay than instrumental responses, the question arises as to how this difference can be theoretically accommodated. We will address this issue within the context of the present experiment: Why is it that the spatial preference tests revealed a general aversion for the black, saccharin, side of the T-maze, while the animals' instrumental behavior failed to reflect this response disposition? All four groups showed a distinct aversion for the black arm on both the Trial 4 and Trial 12 preference tests.
Nevertheless, the animals in Groups U30 and C30 chose the black arm more than they chose the striped arm throughout training, and Groups $\mathrm{U} 1$ and $\mathrm{Cl}$ did not show appropriate instrumental behavior until the second block of four free trials. This lack of correlation between an animal's initial choice response and its subsequent distribution of time in the two arms of the T-maze can be understood in terms of whether an affective response is directly elicited by a conditioned stimulus or occurs in anticipation of that stimulus. The animals spent less time during the preference tests in the black arm of the T-maze than in the striped because, presumably, the former stimulus complex elicited a negative affective response that caused the animal to exit the arm earlier than it otherwise would. On the other hand, for an animal to display appropriate instrumental behavior, the affective response controlled by this stimulus complex must become anticipatory (i.e., occur before the animal enters the black arm), thus discouraging entrance into the black arm. The development of an anticipatory affective response cannot antedate the development of the conditioned affective response itself and may often require many more training trials before it is evident.

The suggestion, then, is that a delayed reinforcer is less effective than an immediate reinforcer in modulating instrumental behavior because the affective response associated with the reinforcer, although perhaps of considerable strength, does not become anticipatory as readily as when the reinforcer is immediate. ${ }^{1}$ The basis of this difference is not clear at present and, without additional empirical data, probably does not warrant speculation. It might be mentioned, however, that the feeble control exerted by a delayed reward on instrumental behavior has been largely attributed to the existence of a substantial preference for an incorrect alternative (D'Amato, Safarjan, \& Salmon, in press); in view of the initial significant preference for the black, saccharin, arm of the T-maze, a similar mechanism might be at work here.

\section{REFERENCE NOTES}

1. Safarjan, W. R., \& D'Amato, M. R. One-trial, long-delay conditioned preference in rats. Manuscript submitted for publication.

2. D'Amato, M. R., \& Cox, J. Long-delay discrimination performance and acquisition in monkeys. Paper presented at the meeting of the Psychonomic Society, St. Louis, Missouri, November 13,1976 .

\section{REFERENCES}

Albent, D. J., \& MAн, C. J. An examination of conditioned reinforcement using a one-trial learning procedure. Learning and Motivation, 1972, 3, 368-388.

Barker, L. M., Best, M. R., \& Domjan, M. (Eds.). Learning mechanisms in food selection. Waco, Tex: Baylor University Press, 1977. 
Best, P. J., Best, M. R., \& Henggeler, S. The contribution of environmental non-ingestive cues in conditioning with aversive internal consequences. In L. M. Barker, M. R. Best, \& M. Domjan (Eds.), Learning mechanisms in food selection. Waco, Tex: Baylor University Press, 1977.

Chen, J., \& Amsel, A. Recall (versus recognition) of taste and immunization against aversive taste anticipation based on illness. Science, 1980, 209, 831-833.

D'Aмato, M. R., \& Buckiewicz, J. Long delay, one-trial conditioned preference and retention in monkeys (Cebus apella). Animal Learning \& Behavior, 1980, 8, 359-362.

D'Aмato, M. R., \& Cox, J. K. Delay of consequences and shortterm memory in monkeys. In D. L. Medin, W. A. Roberts, \& R. T. Davis (Eds.), Processes of animal memory. Hillsdale, N.J: Erlbaum, 1976.

D'Amato, M. R., Safarjan, W. R., \& Salmon, D. Long-delay conditioning and instrumental learning: Some new findings. In N. E. Spear \& R. R. Miller (Eds.), Information processing in animals: Memory mechanisms. Hillsdale, N.J: Erlbaum, in press.

Galef, B. G., JR., \& Osbonne, B. Novel taste facilitation of the association of visual cues with toxicosis in rats. Journal of Comparative and Physiological Psychology, 1978, 92, 907 916.

LETT, B. T. Long delay learning in the T-maze. Learning and Motivation, 1975, 6, 80-90.

LETT, B. T. Long-delay learning of a black-white discrimination:
Effect of varying the length of delay. Bulletin of the Psychonomic Society, 1978, 12, 307-310.

LETT, B. T. Taste potentiates color-sickness associations in pigeons and quail. Animal Learning \& Behavior, 1980, 8, 193-198.

Riesen, A. H. Delayed reward in discrimination learning by chimpanzees. Comparative Psychology Monographs, 1940, 15, $1-54$.

Roвerts, W. A. Failure to replicate visual discrimination learning with a 1-min delay of reward. Learning and Motivation, 1976, 7, 313-325.

WoLfE, J. B. The effect of delayed reward upon learning in the white rat. Journal of Comparative Psychology, 1934, 17, 1-21.

\section{NOTE}

1. Chen and Amsel (1980) recently reported that rats trained to run to flavored water in a runway and then subjected to a single (immediate) pairing of the flavored water and lithium chloride reduced their running speeds on the very next trial; that is, an anticipatory affective response was established by a single poisoning experience. However, the dosage of lithium chloride employed by these investigators was about 15 times that used in the present study.

(Received for publication September 20, 1980; revision accepted January 21,1981 .) 